Enterococcus faecalis surface protein Esp in the pathogenesis of ascending urinary tract infection. Infect Immun 2001;69:4366-4372.

\section{Risk Factors for ICU Infection}

Appelgren and coinvestigators from the Karolinska Hospital, Stockholm, Sweden, conducted a study to identify risk factors for nosocomial infection in the intensive care setting and to provide a basis for allocation of resources. The study was a long-term prospective incidence study of risk factors for nosocomial infection in the surgical-medical intensive care unit of a university hospital.

A total of 2,671 patients were admitted during 4 years, and 562 of 574 patients staying $>48$ hours were observed during 4,921 patient-days (median length of stay 5 days; range, 2-114). Of these, 196 patients (34\%) had 364 nosocomial infections after the median 8 to 10 days, an infection rate of 14 of 100 admissions. Infection prolonged length of stay 8 to 9 days and doubled the risk of death. The infections were $17 \%$ bloodstream infections, $26 \%$ pneumonias, $34 \%$ wound infections, $10 \%$ urinary tract infections, and $13 \%$ other infections. The incidence of bloodstream infection declined significantly during the study years, from $12 \%$ to $5 \%$. In multiple regression analysis, the important variables for infection were central venous catheter, mechanical ventilation, pleural drainage, and trauma with open fractures.

High age, immunosuppression, and infection on admission did not influence the risk of acquiring infection. Trauma patients constituted $24 \%$ of the study population. Trauma with open fractures increased the risk of infection more than twofold $(P=.003)$, mainly due to wound infections.

The authors concluded that trauma patients with open fractures were the patients most at risk of infection, despite low disease severity scores. Resources to prevent nosocomial infection should be allocated to these patients.

FROM: Appelgren P, Hellstrom I, Weitzberg E, Soderlund V, Bindslev L, Ransjo U. Risk factors for nosoco- mial intensive care infection: a long-term prospective analysis. Acta Anaesthesiol Scand 2001;45:710-719.

\section{Staphylococcus epidermidis Biofilms}

The coagulase-negative staphylococci, in particular, Staphylococcus epidermidis, have emerged as major nosocomial pathogens associated with infections of implanted medical devices. These organisms, which are among the most prevalent bacteria of the human skin and mucous membrane microflora, present unique problems in the diagnosis and treatment of infections involving biofilm formation on implanted biomaterials. Epidemiological data that address whether invasive $S$ epidermidis strains can be traced to commensal organisms or an endemic occurrence of distinct strains with enhanced virulence have important implications for the implementation of appropriate infection control measures.

An extracellular polysaccharide adhesin represents a key virulence determinant in $S$ epidermidis and is required for biofilm formation. Production of this adhesin, which is encoded by the ica operon, is subject to phase variable regulation (ON $<>$ OFF switching). Recent advances in understanding the molecular events controlling polysaccharide adhesin synthesis and the potential clinical implications of its phase variable regulation have been outlined and discussed by O'Gara and Humphreys from Dublin. Further research in this area may contribute to the development of novel strategies for therapeutic intervention.

Finally, in addition to antibiotic prophylaxis, preventive strategies to control $S$ epidermidis medical-device-related infections are focusing on the development of improved biomaterials and physical electrical barriers to impede bacterial colonization.

FROM: O'Gara JP, Humphreys H. Staphylococcus epidermidis biofilms: importance and implications. $J$ Med Microbiol 2001;50:582-587. 